\title{
EL DERECHO DE PROTECGIÓN DE DATOS PERSONALES
}

THE RIGHT TO PROTECT PERSONAL DATA

Christian Alberto Arellano López

Titular de la Unidad de Transparencia del Instituto de Becas y Crédito Educativo del Estado de Sonora

Contacto: chrisarellano23@hotmail.com

(D)https://orcid.org/0000-0002-4993-1231

DOI https://doi.org/10.36796/biolex.v0i23.194

Recibido: 30.10.2020/Aceptado: 20.11.2020

\begin{abstract}
RESUMEN
\end{abstract}
En el documento se plasma la nueva dimensión otorgada a los datos personales, a partir de diversas reformas constitucionales y disposiciones legales relacionadas con los sistemas locales y nacional en materia de transparencia y protección de datos personales, que responsabiliza a los sujetos obligados, a transitar de un concepto de Gestión de Datos Personales (GDP) al de un Sistema de Gestión de Protección de Datos Personales (SGPDP), integrado a las políticas de la transparencia, acceso a la información, derecho a la verdad, privacidad, intimidad, autodeterminación informativa, libertad personal, dignidad humana, interés público, límites al estado y memoria colectiva, lo que conlleva obligaciones de carácter humano, materiales, administrativas, tecnológicas y de estructura organizacional de alto nivel.

Datos personales, intimidad, protección, nuevas tecnologías, reconocimiento.

PALABRAS CLAVE

ABSTRACT

The document reflects the new dimension given to Personal Data, based on various constitutional reforms and legal provisions related to local and national systems on transparency and personal data protection, which subjects, to move from a Concept of Personal Data Management (GDP) to a Personal Data Protection Management System (SGPDP), integrated into the policies of transparency, access to information, right to truth, privacy, information self-determination, personal freedom, human dignity, public interest, limits to state and collective memory, which entails obligations of a nature materials, administrative, technological and high-level organizational structure.

Data, Personal privacy, protection, new technologies, recognition.

KEYWORDS 


\begin{abstract}
Sumario
Consideraciones previas | Introducción | Antecedentes del marco jurídico del derecho a la protección de datos personales | El derecho fundamental a la protección de datos personales | Conclusión | Referencias bibliográficas
\end{abstract}

\title{
Consideraciones previas
}

$\mathrm{H}$ oy en día la información es poder y en los últimos años se han creado numerosas empresas especializadas en la recopilación de todo tipo de datos personales; en su gran mayoría para fines comerciales. Lo preocupante de la situación es la inseguridad con que manejan estas empresas, las cuales se encargan de administrar o sustraer estos datos poniendo en peligro incluso la integridad de las personas.

En este sentido, la ley impone a los particulares: personas físicas y morales de carácter público y privado, que realicen el tratamiento de datos personales una serie de obligaciones y reglas para asegurar su uso y protección adecuados.

Para ello debe existir un pilar básico y fundamental de un efectivo sistema integral de protección de datos personales basado en la garantía de la seguridad de la información, entendida ésta como la implementación de medidas administrativas, físicas y técnicas eficaces para garantizar y velar por la integridad, confidencialidad y disponibilidad de tus datos personales. Ello contribuye a minimizar los riesgos de acciones en contra de la información personal como robo, alteración o modificación, pérdida total o parcial, transmisiones indebidas o ilícitas, accesos no autorizados y robo de identidad, entre otras, que pueden lesionar los derechos o libertades de los titulares.

De tal forma que el responsable, el personal externo y el personal interno de los sujetos obligados tengan una mayor tendencia a tomar acciones como: elaborar inventarios de datos personales y de los sistemas de tratamiento.

Pues está comprobado que estos prestan poca atención a capacitar al personal que efectúe el tratamiento de datos personales. (responsable $5 \%$, personal externo $1 \%$ y personal interno $7 \%$ ).

Datos que establecen que mientras el personal externo a los sujetos obligados toma interés alto en el establecer las medidas de seguridad aplicables a los datos 
personales e identificar aquéllas implementadas de manera efectiva con $22 \%$, el responsable muestra un interés del $3 \%$ y el personal interno $5 \%$.

Con lo anterior, podemos deducir que los responsables carecen de los conocimientos adecuados para aplicar la Ley General de Protección de Datos Personales en Posesión de Sujetos Obligados. Es así, que sería conveniente trabajar un esquema con diversos tópicos que nos permita delinear nuevos paradigmas para la protección de datos personales, materia de otro trabajo, que estén a su vez alineados a los parámetros instaurados tanto en el acuerdo mediante el cual se aprueban los parámetros de mejores prácticas en materia de protección de datos personales del sector público, así como lo establecido en el acuerdo mediante el cual se aprueban las disposiciones administrativas de carácter general para la elaboración, presentación y valoración de evaluaciones de impacto en la protección de datos personales.

\section{Introducción}

Los orígenes de la protección de los datos personales según diversos estudios y estudiosos los podemos situar a partir de la sociedad de la información, donde con la industrialización del mundo de los años setenta se vinieron a convertir en parte de la política pública que requirió ser adoptada por el gobierno frente al creciente uso de las tecnologías de la información; En este sentido, las tecnologías vinieron a revolucionar con gran capacidad de producir riqueza a partir del uso de la información personal, lo que hoy en día conocemos como tratamiento de datos personales.

Por otro lado, con el uso de dichas herramientas tecnológicas se evidenció que los gobiernos podían utilizarlas para invadir la vida privada de los ciudadanos y ejercer un mayor control sobre ellos, lo cual trajo consigo no solo la invasión de la vida privada sino de la propia intimidad personal de los individuos.

Con el propósito de conciliar estas dos necesidades opuestas, fue necesario que tanto la sociedad civil organizada y el gobierno, en un ejercicio de participación ciudadana único en su tipo, propusieran la emisión de las primeras leyes de protección de datos personales.

Estas leyes han tenido como objetivo los siguientes ejes rectores: Por un lado el establecer reglas orientadas al tratamiento o utilización de los datos personales por organizaciones públicas y privadas y por el otro, proteger a la persona respecto al uso de su información personal. 


\section{Antecedentes del marco jurídico del derecho a la protección de datos personales}

Etapas relevantes en la evolución de la protección de datos personales en México

1. Declaración Universal de los Derechos Humanos (10 de diciembre de 1948): Contiene el reconocimiento del derecho a la vida privada de las personas; con esto, el derecho humano a la Protección de Datos Personales es reconocido a nivel internacional. Este derecho es subjetivo, autónomo y de tercera generación, el cual garantiza la libertad del individuo en el seno de una sociedad democrática.

2. Avance informático y tecnológico (1 de diciembre de 1980): $\mathrm{Su}$ consecuencia fue el empleo intensivo de información, especialmente en México, donde la legislación ha evolucionado rápidamente.

3. Promulgación de la Ley Federal de Acceso a la Información Pública Gubernamental (11 de julio de 2002): Incluyó la previsión general respecto a la posesión de datos del sector público (consentimiento, información, seguridad, calidad, etc.), así como sus debidas disposiciones.

4. Lineamientos de protección de datos personales (30 de septiembre de 2005): Considera como obligaciones para los entes públicos federales: obtener el consentimiento, informar los propósitos del tratamiento y adoptar las medidas técnicas necesarias para garantizar la seguridad de los datos personales.

5. Plan Nacional de Desarrollo (1 de enero de 2007): Mismo que prevé la necesidad de contar con una Ley Federal que garantice la protección de datos personales en posesión de los particulares, tomando como base lo dispuesto en los principios y tratados internacionales.

6. Reforma al artículo 73 constitucional (27 de marzo de 2007): Que tenía como objeto el dotar de facultades al Congreso Federal para la legislación de protección de datos en posesión de los particulares. 
7. Reforma al artículo 16 constitucional (25 de noviembre del 2008): El cual adiciona un párrafo con la finalidad de reconocer el derecho a la protección de datos personales como una garantía fundamental y autónoma.

8. Reformas constitucionales (30 de abril de 2009): A los artículos 16 y 73 constitucional, y que otorgó el reconocimiento pleno a la protección de datos personales como un derecho fundamental y autónomo.

9. Ley Federal de Protección de Datos Personales en Posesión de Los Particulares (5 de julio de 2010): Promulgada para que los datos personales también sean protegidos por el sector privado.

10. Ley General de Protección de Datos Personales en Posesión de Sujetos Obligados. (6 de enero del 2017): Reglamentaria de los artículos 6, base A y 16, segundo párrafo, de la Constitución Política de los Estados Unidos Mexicanos, en materia de protección de datos personales en posesión de sujetos obligados.

\section{Principales instrumentos internacionales que configuran el derecho a la protección de datos personales}

1. Directrices de la OCDE (1980).

2. Resolución 45795 de la ONU (1980).

3. Convenio 108 (1981).

4. Directiva 957467CE (1995).

5. Marco de privacidad de APEC (1999).

6. Carta de derechos fundamentales de la Unión Europea (2000).

7. Directrices de amortización de la Red Iberoamericana (2007).

8. Estándares internacionales o Resolución de Madrid (2009).

\section{El derecho fundamental a la protección de datos personales}

En virtud de que los datos personales constituyen nuestra información personal más apreciable, misma que nos sirve para llevar a cabo las actividades cotidianas, y que a la vez revela datos privados sobre la personalidad, como son las preferencias o gustos de una persona, entre otros, por estas razones es menester velar por su seguridad y confidencialidad, pero sobre todo porque constituye un derecho fundamental protegido constitucionalmente, el cual nos 
otorga a las personas la facultad de controlar nuestros datos personales, disponer y decidir sobre esos datos y su uso; es por ello, que el objetivo que se establece en la normativa es precisamente el proteger la privacidad y la intimidad de las personas, así como, dar transparencia y certidumbre al tratamiento de los datos, asegurar que los datos que se tratan son correctos y están al alcance sólo de quienes deben utilizarlos para unas finalidades determinadas, es decir, que se cumpla con los pilares fundaméntateles de la protección de datos personales representados a través de sus principios y deberes.

Por ello, resulta sumamente importante proteger los datos personales frente a la sociedad de la información en la que vivimos, en la cual el uso de tecnologías de la información (TIC's) permite comunicar los datos de manera masiva, prácticamente ilimitada, donde estos reflejan quién eres, tus gustos, tu economía, tu salud, tus preferencias, es decir, nos convertimos, como algunos lo llaman, en "seres de cristal".

Es en este sentido, cobra vital importancia el fenómeno del tratamiento de datos personales, sobre todo a través del Big Data ${ }^{1}$, que supone la gestión y tratamiento de inmensas cantidades de datos personales que, si bien es cierto, puede brindar grandes ventajas y beneficios a las organizaciones públicas, las privadas y a la sociedad en general, también puede conllevar ciertos riesgos en materia de privacidad. Es así, que es substancial que cada entidad asiente personal interés en cumplir, entre otros, con el principio de calidad de los datos personales utilizados, así como, en la conservación y almacenamiento de estos.

En esta tesitura, es sustancial difundir las mejores prácticas para el desarrollo social, que abonarán a la implementación de los resultados, acciones de monitoreo y evaluación, tanto en la iniciativa privada como en el sector público, esto vendrá a ser parte de un proceso de aprendizaje institucional que permita a las dependencias tener un modelo de lecciones aprendidas, es decir, un punto de referencia institucional que les muestra que es factible mejorar su propio desempeño y lograr resultados garantizando la rendición de cuentas, la transparencia y la medición continua de los resultados e impacto.

Por lo que respecta a la situación actual en México, pudiéramos agregar que al menos las normas en el contexto nacional han favorecido a la edificación del derecho de protección de datos personales, y consecuentemente a las obligaciones

\footnotetext{
${ }^{1}$ Elena Gil, Big data, privacidad y protección de datos. Madrid: Agencia española de protección de datos. 2016. https://www.aepd.es/sites/default/files/2019-10/big-data.pdf.
} 
directas para el sector público y privado, que, como parte de sus procesos, traten información.

De tal suerte, que las disposiciones en materia de protección de datos personales se fundamentan principalmente en dos ordenamientos: el primero, para el sector privado que corresponde a la Ley Federal de Protección de Datos Personales en Posesión de Particulares (LFPDPPP) y de aplicación federal; el segundo, para el sector público que concierne a la Ley General de Protección de Datos Personales en Posesión de Sujetos Obligados (LGPDPPSO) y de aplicación en los tres órdenes de gobierno (Federal, Estatal y Municipal). No obstante, existen excepciones sectoriales para la aplicación de estas dos leyes.

Es importante enfatizar que el modelo mexicano es un híbrido, consecuencia de la asociación de la visión europea y de algunos elementos del derecho anglosajón. Esto, toda vez que la protección de datos personales en nuestro país se exalta al valor de un derecho humano, pero también reconoce esquemas de autorregulación y legislación sectorial, por lo cual, se hace más complejo su cumplimiento.

Así pues, según los datos recabados en el denominado "Estudio de protección de datos personales entre usuarios y empresas" que presentará la Asociación Mexicana de Internet, mismo al cual hace referencia la autora Olivia Andrea Mendoza Enríquez, del Centro Público de Investigación e Innovación en Tecnologías de la Información y Comunicación (INFOTEC), proporciona una noción de los problemas y retos en el cumplimiento de la Ley Federal de Protección de Datos Personales en Posesión de los Particulares y nos permite establecer los principales desafíos en torno al mismo ${ }^{2}$, ese similar referente se puede inferir al sector público, derivado del Tercer Censo Nacional de Transparencia, Acceso a la Información Pública y Protección de Datos Personales en los ámbitos Federal y Estatal, que presentó recientemente el Instituto Nacional de Estadísticas y Geografía (INEGI), a través de su comunicado de prensa número $655 / 19$, de fecha 11 de diciembre del $2019 .^{3}$

En este sentido, el $44 \%$ de los sujetos obligados evaluados no poseen el conocimiento necesario sobre la Ley Federal de Protección de Datos Personales en Posesión de Particulares (LFPDPPP), tampoco de la Ley General de Protección

\footnotetext{
${ }^{2}$ Olivia Mendoza, "Marco jurídico de la protección de datos personales en las empresas de servicios establecidas en México: desafíos y cumplimiento". Revista IUS, 41 (2018): 267-291. http://www.scielo.org.mx/pdf/rius/v12n41/ 1870-2147-rius-12-41-267.pdf.

${ }^{3}$ Instituto Nacional de Estadísticas y Geografía, Tercer censo nacional de transparencia, acceso a la información pública y protección de datos personales en los ámbitos federal y estatal. https://www.inegi.org.mx/ contenidos/saladeprensa/boletines/2019/EstSegPub/CNTAIPPDP_2019.pdf.
} 
de Datos Personales en Posesión de Sujetos Obligados (LGPDPPSO), ni que hablar de los esquemas de autorregulación. En relación con el ejercicio de derechos ARCO, $50 \%$ de los sujetos obligados evaluadas no tiene el conocimiento necesario para su atención y, por lo tanto, podrían ser acreedoras a una sanción por el incumplimiento a las disposiciones de la LFPDPPP. ${ }^{4}$

En el mismo tenor, $30 \%$ de los sujetos obligados no conocen las acciones que han emprendido para realizar el cumplimiento de la ley, $48 \%$ han emprendido acciones de capacitación de personal dentro de su organización y, del resto, $20 \%$ han contratado a una empresa legal especializada en esos temas, sólo $6 \%$ ha contratado a una persona especializada en la ley. No obstante, $50 \%$ de los sujetos obligados considera que el cumplimiento de la Ley genera gastos adicionales. ${ }^{5}$

Queda por reflexionar cuáles son las limitaciones de este derecho a la privacidad, y qué correcciones pueden ser concedidas para la aplicación del derecho. Establecer antes de la experiencia la línea exacta entre la dignidad y la conveniencia del individuo, y hasta donde debe ceder a las demandas del bienestar público o de la justicia privada.

\section{Conclusión}

Aunque se tenga la recientemente aprobada, publicada y retocada Ley General para la Protección de Datos Personales en Posesión de Sujetos Obligados y la de Particulares, donde se "garantiza" la protección jurídica de los derechos de las personas (derechos ARCO), no se considera segura ante la agresividad de la informática, pues siempre estará presente el factor económico ante los intereses del mercado, que influyen mucho para la intromisión y violación de la privacidad e intimidad de datos personales, por lo que, se requiere de trabajar en este sentido alguna propuesta para aplicarla desde nuestro entorno y de ser viable como una mejor práctica para el sector público.

\section{Referencias bibliográficas}

Agencia Española de Protección de Datos (AEPD) y a la Asociación Española para el Fomento de la Seguridad de la Información. Código de buenas prácticas en protección de datos para proyectos big data. España. 2019.

\footnotetext{
${ }^{4}$ Olivia Mendoza, "Marco jurídico de la protección de datos personales en las empresas de servicios establecidas en México”, p. 279.

${ }^{5}$ Olivia Mendoza, "Marco jurídico de la protección de datos personales en las empresas de servicios establecidas en México”, p. 279.
} 
https://www.aepd.es/media/guias/guia-codigo-de-buenas-practicas-proyectosde-big-data.pdf.

Colegio de Contadores Públicos de México AC, "La importancia de la protección de datos personales”. Excelsior. 23 de enero de 2012. https://www.excelsior.com.mx /opinion/2012/01/23/colegio-de-contadores-publicos-de-mexico-ac/804414.

García, Aristeo. "La protección de datos personales: derecho fundamental del siglo XXI. Un estudio comparado”. Boletín Mexicano de Derecho Comparado, 120 (2007). http://www.scielo.org.mx/pdf/bmdc/v40n120/v40n120a3.pdf.

Gil, Elena, Big data, privacidad y protección de datos. Madrid: Agencia española de protección de datos. 2016. https://www.aepd.es/sites/default/files/2019-10/bigdata.pdf.

Instituto Nacional de Estadísticas y Geografía, Tercer censo nacional de transparencia, acceso a la información pública y protección de datos personales en los ámbitos federal y estatal. https://www.inegi.org.mx/ contenidos/saladeprensa/boletines/2019/EstSegPub/CNTAIPPDP_2019.pdf.

Instituto Nacional de Transparencia, Acceso a la Información y Protección de Datos Personales, Introducción a la Ley General de Protección de Datos Personales en Posesión de Sujetos Obligados. Primera Edición. Ciudad de México, 2017. http://www.inaes.gob.mx/doctos/pdf/transparencia/manual\%20LGDPPSO.pdf.

Mendoza, Olivia, "Marco jurídico de la protección de datos personales en las empresas de servicios establecidas en México: desafíos y cumplimiento". Revista IUS, 41 (2018): 267-291. http://www.scielo.org.mx/pdf/rius/v12n41/18702147-rius-12-41-267.pdf.

México:

Comisiones Unidas de Gobernación, y de Estudios Legislativos Primera, Dictamen de las Comisiones Unidas de Gobernación y de Estudios Legislativos Primera, con Proyecto de Decreto por el que se expide la Ley General de Protección de Datos Personales en Posesión de Sujetos Obligados.http://www.senado.gob.mx/comisiones/gobernacion/docs/proteccion _datos/Documento3.pdfMéxico. Congreso Constituyente. Constitución Política de los Estados Unidos Mexicanos. Diario Oficial, 24 de diciembre de 2020. http://www.diputados.gob.mx/LeyesBiblio/ref/cpeum.htm.

Congreso General de los Estados Unidos Mexicanos. Ley Federal De Protección de Datos Personales en Posesión de Particulares. Diario Oficial, 05 de Julio de 2010. http://inicio.ifai.org.mx/LFPDPPP/LFPDPPP.pdf.

México. Secretaria de Gobernación:

Acuerdo mediante el cual se aprueban las disposiciones administrativas de carácter general para la elaboración, presentación y valoración de evaluaciones de impacto en la protección de datos personales. 23 de enero de 
2018. http://www.dof.gob.mx/nota_detalle.php?codigo $=5511113 \& f e c h a=23 / 01 / 2$ 018.

Acuerdo mediante el cual se aprueban los Parámetros de Mejores Prácticas en Materia de Protección de Datos Personales del Sector Público. 09 de octubre de 2019. https://dof.gob.mx/nota_detalle.php?codigo=5574878\&fecha=09/10/2019.

Acuerdo mediante el cual se aprueban las disposiciones administrativas de carácter general para la elaboración, presentación y valoración de evaluaciones de impacto en la protección de datos personales. 23 de enero de 2018. http://www.dof.gob.mx/nota_detalle.php?codigo $=5511113 \& f e c h a=23 / 01 /$ 2018 .

Pulido, Miguel "Convergencias y divergencias: acceso a la información y la tutela de los datos personales”, en Retos de la Protección de los Datos Personales, editado por Instituto de Acceso a la Información Pública y Protección de Datos Personales del Distrito Federal, pp. 79 - 102., 2011. http://docplayer.es/7114112Www-infodf-org-mx-2011-instituto-de-acceso-a-la-informacion-publica-yproteccion-de-datos-personales-del-distrito-federal.html. 
Cuaderno Jurídico y Político y Revista de Ciencias Jurídicas y Políticas son revistas académicas de publicación semestral del Instituto Centroamericano de Estudios Jurídicos y Políticos ICEJP y la Escuela de Ciencias Jurídicas y Políticas respectivamente.

BIOLEX Revista Jurídica del Departamento de Derecho es una publicación semestral por la Universidad de Sonora, a través de la División de Ciencias Sociales, Departamento de derecho.

BIOLEX incorporada en:

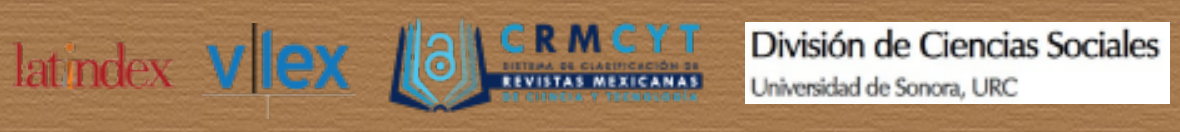

CJP y RevistaCJP incorporada en:

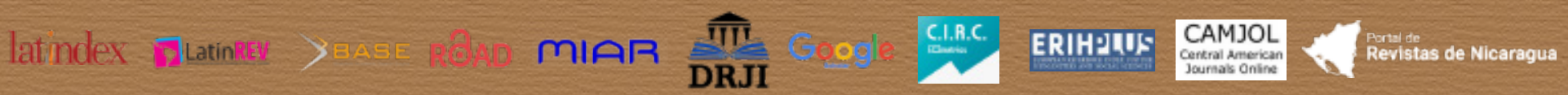

